\title{
Correction to: Trust and Trustworthiness across Cultures
}

\author{
Catherine T. Kwantes and Ben C.H. Kuo
}

\section{Correction to: \\ C. T. Kwantes, B. C. H. Kuo (eds.), Trust and Trustworthiness \\ across Cultures, Springer Series in Emerging Cultural Perspectives in Work, Organizational, and Personnel Studies, https://doi.org/10.1007/978-3-030-56718-7}

This book was inadvertently published without updating the following corrections in the chapters. The book has been updated with these changes.

Page 4, line 6: The sentence should read as "For example, in an early work"

Page 4, line 27: The sentence should read as "and how trustworthiness is determined based on both in-role and extra-role behaviors (Kwantes, 2019)."

Page 5, last line: The sentence should read as "He notes that when individuals conduct themselves according to role expectations and therefore exhibit in-role behaviors, that information is not used by others who view that behavior as expected due to the roles individuals fill - such behavior is prescribed - and therefore offers no insights regarding the person enacting the role. On the contrary, extra-role behaviors, or those behaviors that are beyond what is expected by individuals within a

\footnotetext{
The updated versions of the chapters can be found at https://doi.org/10.1007/978-3-030-56718-7_1 https://doi.org/10.1007/978-3-030-56718-7_2 https://doi.org/10.1007/978-3-030-56718-7_3 https://doi.org/10.1007/978-3-030-56718-7_4 https://doi.org/10.1007/978-3-030-56718-7_7
} 
role, are thought to reflect attributes of those individuals personally and are therefore used to assess the trustworthiness of that individual."

Page 6, line 6: The sentence should read as "We argue, however, that both in-role and extra-role behaviors are important to assessing trustworthiness."

Page 6, line 15: The sentence should read as "individuals may work with those they know as well as those they do not know well at a personal level. Particularly for those they do not know well, in-role performance and extra-role performance become internal metrics for a person's trustworthiness."

Page 20, line 5 from bottom: The sentence should read as "With regard to family, $92.8 \%$ of the participants reported they would trust somewhat or completely; people you know personally, $57.8 \%$ reported they would trust somewhat or completely; neighborhood, $44.2 \%$ reported they would trust somewhat or completely; and only $18 \%$ reported they would trust people you meet for the first time somewhat or completely."

Page 24, line 4: The sentence should read as "However, focusing."

Page 33, line 15: In the abstract section, the sentence should read as "Craig (2017) sees trust as human nature and that trust can also be learned and unlearned because when trust is betrayed (more than once), then caution sets in."

Page 33, line 17: In the abstract section, the sentence should read as "Hence the proverb 'once bitten, twice shy' is known to famously have used the version of the saying which implies "unpleasant experience induces caution."

Page 36, last line: The sentence should read as "While caution and distrust are not the two opposites of trust, both can coexist (Lewicki\&Brinsfield, 2009)."

Page 45, line 2: The comma has been removed and this should read as "(2011) showed."

Page 66, line 15: The comma has been included and the text should read as "In contrast, political trust."

Page 134, line 20: The last sentence of the summary should read as "an introduction to trust in Canada." 